Document downloaded from:

http://hdl.handle.net/10251/164235

This paper must be cited as:

Garcia, E.; Araujo, A.; Palanca Cámara, J.; Giret Boggino, AS.; Julian Inglada, VJ.; Botti, V. (2019). Requirements for an Intelligent Maintenance System for Industry 4.0. Springer. 340351. https://doi.org/10.1007/978-3-030-27477-1_26

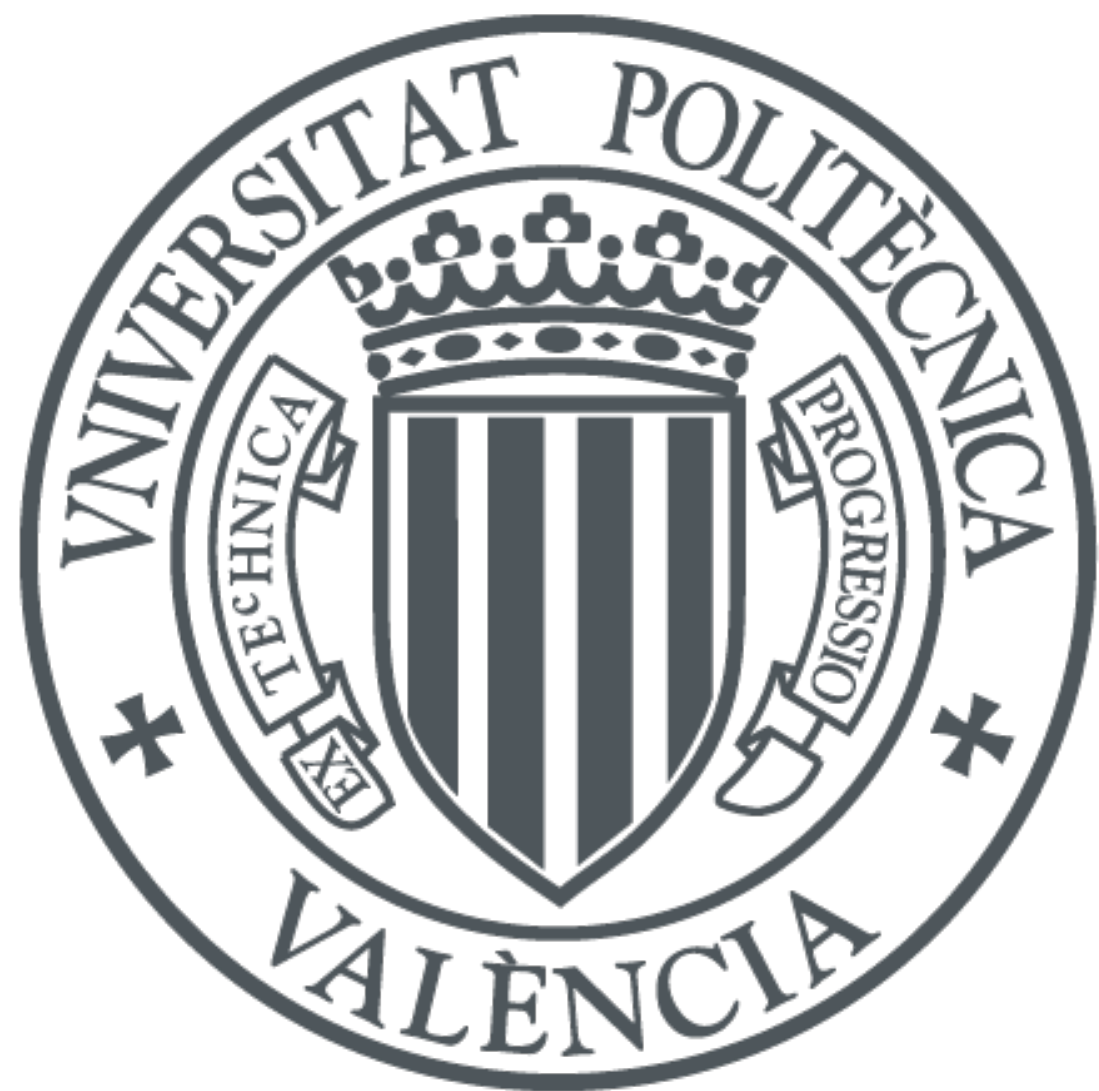

The final publication is available at

https://doi.org/10.1007/978-3-030-27477-1_26

Copyright Springer

Additional Information

comprobación paso "titulo publicación " - Service Oriented, Holonic and Multi-agent Manufacturing Systems for Industry of the Future 


\title{
Requirements for an Intelligent Maintenance System for Industry 4.0
}

\author{
Emilia Garcia, Angelo Costa, Javier Palanca, Adriana Giret, Vicente Julian, \\ Vicente Botti
}

\begin{abstract}
Recent advances in the development of technological devices and software for Industry 4.0 have pushed a change in the maintenance management systems and processes. Nowadays, in order to maintain a company competitive, a computerised management system is required to help in its maintenance tasks. This paper presents an analysis of the complexities and requirements for maintenance of Industry 4.0. It focuses on intelligent systems that can help to improve the intelligent management of maintenance. Finally, it presents a summary of lessons learned specified as guidelines for the design of such intelligent systems that can be applied horizontally to any company in the Industry.
\end{abstract}

\section{Introduction}

Maintenance engineering has always played an important role for the industry performance. European Standards regarding maintenance [1] define maintenance as the "combination of all technical, administrative and managerial actions during the life cycle of an item intended to retain it, or restore it to, a state in which it can perform the required function", while maintenance management is defined as "all the activities of the management that determine the maintenance objectives or priorities, strategies and responsibilities and implement them by means such as maintenance planning, maintenance control and supervision, and several improving the methods including economical aspects in the organization" [3].

From these definitions we can point that maintenance are a set of complex activities that involve the whole manufacturing company's structure and organisations.

Emilia Garcia, Angelo Costa, Javier Palanca, Adriana Giret, Vicente Julian, Vicente Botti Computer Science Department, Universitat Politècnica de València, Camino de Vera s/n, 46022, Valencia, Spain e-mail: mgarciaedsic.upv.es, anardaledsic.upv.es, jpalancal dsic.upv.es, agiretedsic.upv.es, vinglada@dsic.upv.es, vbottiedsic. upv.es 
Taking into account the moment when the maintenance actions are performed, maintenance can be classified as corrective or preventive.

Corrective maintenance actions are executed in response to the failure of a machine. The objective of this kind of maintenance action is the restoration of the production system functionality. These kind of failures usually stop the production. So, besides the cost of the machine's reparation, there are other relevant loses such as the cost of shutdown and the restart the production line, and the possible lose of clients due to the impossibility of meeting the demand. In the current competitive environment this kind of situations can be critical for any industry due to the high level of competitiveness and the required high standards regarding quality and time constrains.

Preventive maintenance tries to avoid failures and reduce production downtime by performing maintenance actions before the failure happens. Traditionally, this kind of maintenance is time-based, i.e., this maintenance is performed on equipment based on a calendar schedule defined by the company responsible for each machine. This kind of maintenance still is the most extended applied method, although it has been proved as flawed and unreliable [6]. Several studies prove that the time between failures not only depends on the specific machine but also on other factors. Many times the maintenance actions performed are not necessary and other times they are planned too late and the failure appears before the execution of the preventive maintenance action.

The last decade developments in the intelligent systems area, the computational power of internet of things and the big data analysis have arisen new possibilities regarding maintenance. Preventive maintenance can be improved by using predictive techniques that predict a failure before it happens taking into account the values of several sensorized parameters and the analysis of previous registered data. Predictive maintenance is crucial for adapting maintenance management systems to the Industry 4.0.

Although the application of predictive maintenance and more specifically the inclusion of intelligent technologies into the maintenance management systems has been proved as very beneficial for the industry, there are still many open challenges regarding this topic and it is not completely implanted in the Industry. One of the efforts is embodied in the concept of digital twin, which is a digital representation of the manufacturing process. The aim is to have a complete digital footprint of the product life cycle, being executed ahead of the actual production, thus being able to preeemptively detect problems.

This paper contributes to the state-of-art by providing (1) an analysis of the most relevant requirements and functionalities that a computerised maintenance management system (CMS) has for the Industry 4.0; (2) a review of current intelligent technology that can improve the maintenance performance; (3) a summary of lessons learned specified as guidelines for the design of intelligent maintenance systems that can be applied horizontally to any company in the Industry.

The rest of the paper is organised as follows: 
- Section 2 analyses the requirements and functionality that are expected in a computerised maintenance management system (CMMS) for an industry that wants to remain competitive in the Industry.

- Section 3 reviews current technology for supporting these requirements focusing mainly in the possibilities that artificial intelligence and big data analysis offer.

- Section 4 presents a set of guidelines for designing an intelligent CMMS that can be applied horizontally to any company in the Industry.

- Finally, Section 5 summarised the main contributions of this paper and the lines of our future work.

\section{Requirements analysis for Maintenance in the Industry 4.0}

In the same way that mechanisation through water and steam power were the key factors for the first industrial revolution, the electricity was the key factor for the second one, the automation and the use of computers were the key factors for the third one [9]. Now, many authors are considering the integration of intelligent systems and algorithms, and the communications between machines and between machines and humans the key factors for the fourth industrial revolution [15].

As is presented in the European standards [1], nowadays maintenance management systems should combine corrective, preventive and predictive maintenance. A complete predictive maintenance is the goal of the Industry 4.0. However, at the moment, predictive maintenance algorithms and systems do not cover the whole spectrum of possibilities that may happen inside a manufacturing company. As is shown in Section 3, the systems available offer solutions to specific problems but in any case a complete solution.

Moreover, in order to manage an optimal and intelligent maintenance, it is necessary the to plan the maintenance actions taking into account other departments of the manufacturing company and also the state of the plant and production orders [14]. Figure 1 summarises the maintenance system's main functionalities and its interactions with other components of the manufacturing company.

The data that the maintenance system receives and analyzes as follows:

- Business objectives: maintenance decisions must be in line with the company's policy and its business objectives of production, human resource management, customer service, etc.

- Human and material resources management system: scheduled and immediate action maintenance tasks after a failure require expert personnel work and the provision of specific materials and raw materials. It is therefore important that the maintenance management system analyzes this data for planning and executing maintenance orders. Depending on the individual business structure of each organisation, either a dedicated maintenance team will be available or resources will have to be requested from other areas of industry, with the associated analysis for these areas. 


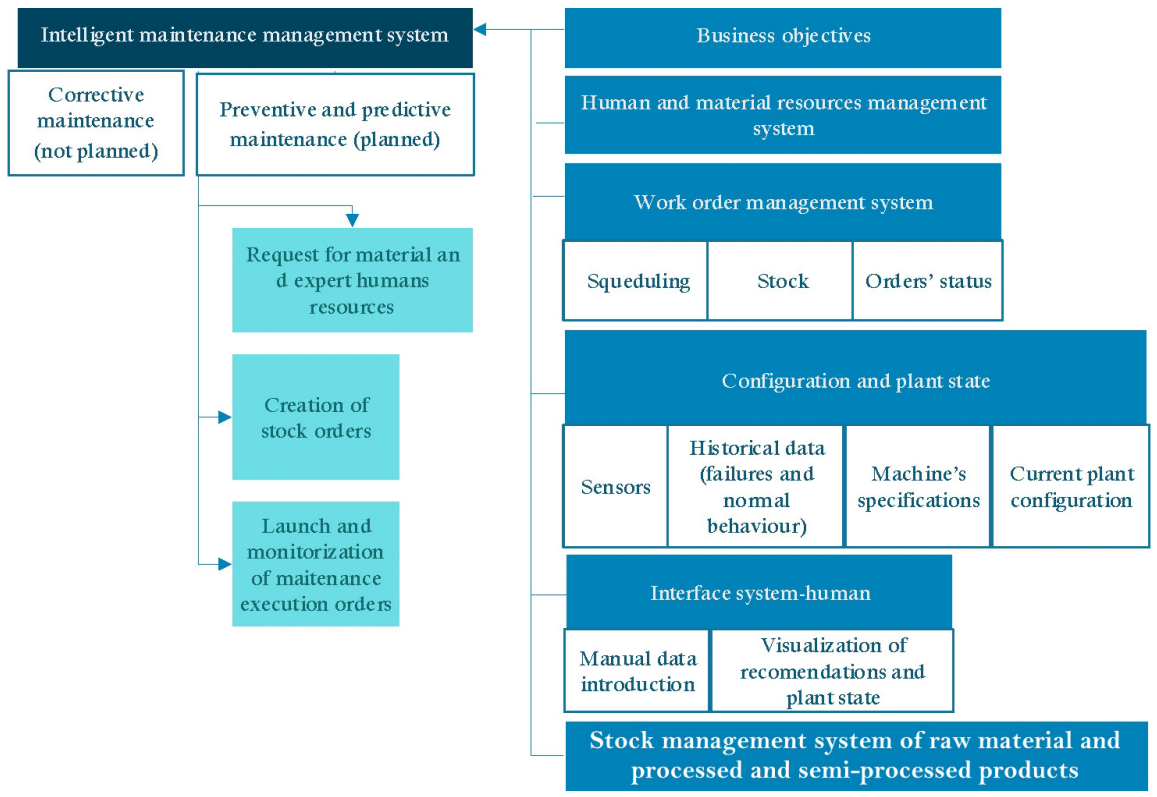

Fig. 1 Management types according to European Standards

- Work order management system: the maintenance system must be able to include work orders for the execution of maintenance tasks or inventory creation to mitigate the consequences of a future maintenance shutdown. In order to carry out this function, it is required to analyse the current work orders. Similarly having access to this data will allow to calculate the costs of a machine breakdown or delay. The maintenance tasks are scheduled taking into account the production orders, their planning, their state of development and their priority.

- Plant configuration and status: this is the main data source of the maintenance management system. The system must have:

1. The specifications of the machines in which the factory recommendations for their correct use and maintenance are found.

2. The configuration of the plant and if there are other possible configurations. With this data the system would be able to recommend a configuration change if the costs of doing so were less than the benefits.

3. Sensorization of the machines and the plant. For the correct execution of the predictive maintenance it is necessary to have real time data that indicates the state of the machinery. The most common sensorization is based on temperatures and vibration levels, however in a horizontal model it can not be specified the type of sensorization as it is fully dependent on domain.

4. Historical data on machine operation and failures. Real-time sensing is only useful if we have a history that allows us to discern which sensed data are 
within the normal range of operation of the machine and when they are an alert to a possible machine failure.

- Human Expert Interaction System: maintenance and production technicians that are in charge of manually and visually monitoring production must be able to interact with the intelligent maintenance management system in order to be able to provide alarm notifications or configuration changes.

- Stock management system for raw materials, processed and semi-processed products: manufacturing companies tend to produce on demand, minimising the costs associated with maintaining stock. This production approach is vulnerable to unforeseen events and is usually drastically affected when corrective or even preventive maintenance has to be carried out. Therefore, on some occasions the intelligent maintenance management system must recommend the creation of a stock that meets the forecasts of customer orders provided by a previous study of prognosis.

These requirements' analysis is fundamental to the design of maintenance management systems and to its successful implementation. They are observable in the next section as state of the art of projects, methods, case studies and concepts related to maintenance in Industry 4.0 are presented and the requirements and solutions outlined.

\section{Review of Intelligent Technology applied to Maintenance}

Recent advances in the development of technological devices and software for Industry 4.0 have pushed the boundaries of the predictive maintenance (PdM) techniques that were used until very recently, resorting to knowledge and developments outside of the usual industry domain. For instance, we now can observe the usage of non-related technologies like Artificial Intelligence (convolutional neural networks, deep learning, machine learning, etc.) and Big Data that are not commonplace in industry parameters. At the same time, traditional procedures still continue, although they are now even more fine tuned and personalised to their usage scenarios, a result of the technological advancements of the sensor systems and computational models.

Classical concepts of PdM are presented by Hashemian and Bean [6] where the authors divide the domain into three parts (according to their data origins) that are: "the existing sensor-based technique, the test-sensor-based technique (including wireless sensors), the test-signal-based technique (including the loop current step response method, the time-domain reflectrometry test, and the inductancecapacitance-resistance test)". They argue that time-based maintenance models are unable to address in-time maintenance procedures. Meaning that only a residual percentage of the problems will be preemptively corrected before machine failure. They propose online detection of near-failure conditions, resorting to sensors systems and simple prediction algorithms. In the same line of work is the one by Crespo and Gupta [3], were they present a framework that covers the complete maintenance 
process. Within this framework they suggest balancing the maintenance procedure using performance indicators that resorts to plans of reliability and improvement, which in turn are designed using data of historic failure and repair procedures. Furthermore, the involvement of upper-management in the process is advised, to foster and select the best repair teams, optimising also the human involvement. Lu et al. [8] present a proactive approach to PdM (still being actively used currently) were a prototype sensor system was used to monitor electrical motors and, using noisecancellation models, they were able to predict bearing failure with relative accuracy (they were able to detect that a high risk of failure preemptively in a case scenario). This work showed that prediction may reduce greatly the maintenance costs by reducing production stoppage times. Since then, most industrial machines are now equipped with large sensors arrays that are able to perceive the most tiny changes in state or environment.

More recently, newer technologies and concepts were introduced that used large amount of data produced by the machines' sensors. This opened the door to advanced PdM models, that are able to accurately forecast the time-to-failure of components critical to the machine operation. This constitutes the Industry 4.0 concept.

Chen et al. [2] present a framework for PdM that uses ontology-based and big data models to create a Smart Factory. This type of factories (and industry) consist in modular and flexible production processes that are easily re-configurable and use just-in-time procedures, accompanying fast changes in demand, thus in production. With these models, the factory is able to predict faults resorting to high volume of experience and incorporating the expert perspective in the decision-making process, improving the knowledge about the faults and repair procedure and time. Furthermore, they introduce the concept of cyber-physical production system, an amalgamation of intelligent sensors, embedded terminal systems, intelligent control system, and communications facilities.

Also using Big Data, the work of Wan et al. [16] proposes an offline prediction algorithm that uses historical data from the sensor arrays, using neural networks. They propose a progressive approach towards maintenance: when a component/machine reaches an "unsafe" threshold, it is calculated the remaining effective working time by analysing the historical data. If the component/machine fails prematurely, this data is used to reconfigure the model. This approach saves time and cost by avoiding premature change of components and stoppage of production. Their results showed an accuracy increase of 15 percent points over traditional methods.

Using machine learning procedures, the work of Paolanti et al. [11] that proposes this technique to create decision trees that are faster and simpler to follow and implement. In their case study they have used a large sample of sensor data (over 530000 of 15 features) and a simple utility function that focused on thresholds. The result was a $95 \%$ accuracy of failure detection, thus improving the maintenance response time.

Using another approach is the work of Ferreira et al. [4] that, unlike most newer implementations, uses short-term forecasting, as the machine since their case study had high variation on failure rates. The operational model follows the same path as the one presented by Wan et al. [16], being structured by thresholds (with an average 
of 150 days) at which the expert advice should be used to verify the machine's condition.

This short sample presents an overview of the developments currently made, using exciting and new technologies. But they are not free from faults and shortcomings that should be addressed. These issues are addressed in section 3.1 where they are outlined and suggestions to tackle them are presented in section 4 .

\subsection{Current Issues}

$\mathrm{PdM}$ is a continuous and complex process that is the result of a set of tasks that define the goals, priorities, strategies and responsibilities that constitute the maintenance process. One common issue of the methods presented in the previous section is the lack of general vision and the tight coupling with their case studies. In some cases they have to be modified severely to be used in other scenarios. For instance, Ferreira et al. concept [4] cannot be used in complex systems with large sensor arrays due to their rather larger deviation in terms of maintenance scheduling.

Although Crespo Marquez and Gupta [3] argues that there is an overall lack of maintenance models, Wan et al.[16] presents a complete model. These models are only very recently being presented and we believe that it will take some time to see them being used in the industry. Some of the reasons were presented a few years back but are still valid, like the ones presented by Goh et al. [5] and Potes et al. [13].

Summarising, these are the most pertinent issues related to maintenance that $I n$ dustry 4.0 is currently facing:

1. Lack of specifications: the diversity of problems associated with maintenance is very broad and is usually specific for each sector, for each company or even for each line of production. Furthermore, the level of complexity increases due to the fact that the interrelationships between the machines and maintenance problems are not usually studied. This results in a lack of protocols and maintenance specifications at the sector's a horizontal and vertical levels.

2. Stocks relevancy: on-demand production has improved the factories' efficiency and profitability. However, the lack of parts means that an unplanned shutdown due to maintenance could potentially produce the non-fulfilment of delivery terms and increase the costs derived from this event.

3. Data scarcity: to be able to reduce the corrective maintenance, or unplanned maintenance, and ceasing production, it is important to attain large amounts of data to be used to create maintenance plans that includes the prediction of failures. While most factories have currently large sensors arrays, most of them are only being used for real-time warnings and not historical collection.

4. Expert knowledge: maintenance employees may not have the appropriate experience and knowledge necessary to plan and evaluate the possible risks of delaying overall maintenance. It is necessary to have digitised intelligent tools that are able to reliably planning avoiding risks and consequences of failures and faults. 
5. Complex technological processes: industries currently integrate processes involving large sequences of pre-processes that involve a large number of complex machinery with specific input and output restrictions. These restrictions are hard to sequence and plan, thus planning tools should be employed to address large computations.

6. No integration: despite the fact that most companies have already begun their process of data digitising, they usually are present in different management systems that are not able to synchronise or coordinate. This causes the prognosis and planning processes to be unnecessarily complicated and requires manual labour to connect systems and synchronise data.

These issues can be tackled with a comprehensive framework that is able to encapsulate horizontal and vertical management together with state of the art technological advancements to predict accurately maintenance faults and create scheduling plans to overcome them, being heterogeneous in terms of the domain so it is applicable to the wide industry.

\section{Designing Intelligent Maintenance Management Systems}

In this section we propose a set of guidelines for the design of intelligent maintenance systems that can be applied horizontally to any company in the industry by a synthesis of the analysis of the state-of-art and the schemes offered by experts.

The design of any maintenance system should include three main steps:

\subsection{Configuration}

Predictive maintenance actions need to be configured taking into account the existing information regarding the machines' specifications, the plant configuration and the study of previous production results and failures [7]. Thus, the first step of a maintenance system should configure the maintenance actions taking into account this knowledge, in order to do that the following steps must be executed:

1. Integration of the historical data: Nowadays the majority of the industries save data during production. This data is usually saved in separated databases in different systems. Besides, most of times the data lacks of tags. That fact obstructs the intelligent an automatic analysis of the data. Therefore, the first step is to select, integrate, filter and tag the data from the different systems [14].

2. Significant parameters: The second step is identify the significant parameters and their range of acceptable values. The machines' specifications and the integrated data obtained in the previous step should be analysed using data mining and artificial intelligent algorithms. This algorithms are guided by the human experts in order to be more efficient. This analysis will produce the following results: 


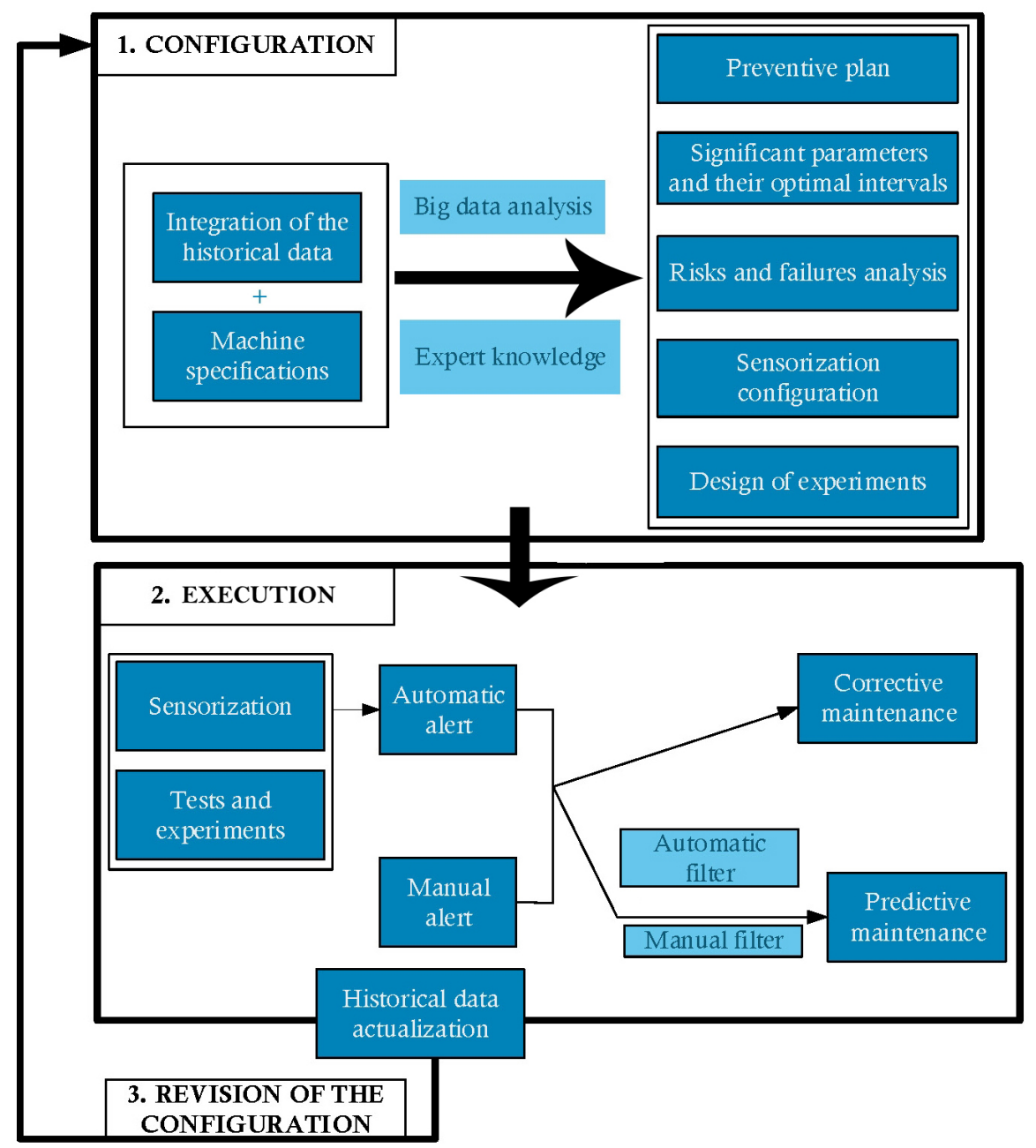

Fig. 2 Designing intelligent maintenance management systems

a. A failure mode analysis (FMA) and a failure mode effects analysis (FME) [12].

b. A selection of the most significant parameters.

c. The specification of the acceptable range of values for each parameter and a recommendation of what to do if the sensorization detects a value out of this range.

d. A preventive maintenance plan: There are few scenarios when preventive planned maintenance is recommended over predictive maintenance. These scenarios are usually related to the lack of data in a specific area that obstruct the definition of a trustworthy predictive maintenance plan. 
3. Reconfiguration of the sensorization: Nowadays most of the industries have some sensors to collect data about the production process and the state of the machinery. However, the previous analysis usually shows the need of adding and the possibility of removing sensors. The need of adding sensors is usually related to the lack of data of an already known significant parameter. Another scenario where more sensors are needed is when a failure cannot be explained with the already detected significant parameters. The possibility of removing a sensor occurs when that data analysis proves that a parameter is not significant.

4. Experiments design: There are some parameters than cannot be directly measured during the production process because they need to be measured after performing an action on the product. For example in the wood sector is common to perform a inflation test consisting of sinking the wood in water and measure the level of inflation. The data obtained in these experiments will be integrated with the sensorized parameters to be able to discover correspondences and associations.

As is shown in previous sections, currently predictive maintenance do not cover the whole spectrum of possibilities. Therefore, an analysis of the critical points of the machinery and plants that are not covered by predictive maintenance must be done in order to configure time-based preventive maintenance actions.

\subsection{Execution}

Intelligent maintenance systems should monitor and control, in real time, the production of orders as well as the quality of the products produced and the state of the machinery [10]. In order to handle with failures and to avoid failures in real time the maintenance system should include the following functionalities:

1. Sensorization: It is the process of collecting the data related to the production in real time. This data must be saved after a post-process in which it is tagged and integrated with previous data and data of other systems. This data will increase the historical data database. The constant analysis of this data allows to generate alerts when a significant parameter is out of his range.

2. Experimentation and tests: Following the design of experiments established in the configuration phase, the tests are executed obtaining data that, as well as the data from the sensorization, must be saved after a post-process in which it is tagged and integrated with previous data and data of other systems, will increase the historical data database and generate alerts when a significant parameter is out of his range.

3. Manual alert: The maintenance experts must have an interface that allows them to interact directly with the intelligent maintenance system in order to create manually an alert demanding a immediate or differed maintenance action, to react and monitor automatically created alerts and to add information to the predictive system to estimate the risks of doing or not doing a specific action. 
4. Corrective maintenance: If a failure is detected the system should include an immediate maintenance action to restore the production. The data failure will be tagged in the historical data for improving the predictive maintenance algorithm.

5. Predictive maintenance: When an out of range value is observed in a significant parameter a new maintenance action is scheduled. Depending on the FMA and the FME the action will be scheduled to be executed immediately or it will be programmed taking into account the business goals, current state of the the production orders and the general state of the plant.

\subsection{Reconfiguration}

Maintenance actions and processes are not static. They should be reconfigured cyclically in order to optimise the production. It is necessary to cyclically review the validity of the sensor configuration, the selection of significant parameters, their acceptable ranges of values, and the necessary experiments and tests.

The frequency of this configuration review depends on each plant on the rate in which new data is generated because only if there is a relevant quantity of new data, changes in the results of the intelligent algorithms applied will be observed.

Besides, it is important that the maintenance management system analysises the evolution of the mean time between failures (MTBF) in order to launch a reconfiguration if there is a significant change in this value.

\section{Conclusions and Future Work}

This paper presents guidelines to design intelligent maintenance systems, through specific non-domain-centric steps that are generic and easy to extrapolate to any company. The goal is to address the necessities of the industry, producing a toplevel framework that combined with novel technologies is able to produce robust and efficient PdM systems.

This paper is the result of the initial developments made in the Platinum project, whose objective is to propose maintenance solutions that increase equipment availability and lower costs and faults associated to maintenance tasks. The additional goal of this project is to produce a generic platform that can be implemented in any company, and using industry-standard tools and sensor arrays produce PdM models that give way to intelligent maintenance systems.

In terms of future work, the next step is to implement the FIWARE framework ${ }^{1}$ with machine learning modules in a real case study scenario at the Sonae Araucd ${ }^{2}$ project partner company.

\footnotetext{
${ }^{1}$ https://www.fiware.org/

${ }^{2}$ https://www.sonaearauco.com/es/
} 
Acknowledgements This work is supported by the FEDER/Ministry of Science, Innovation and Universities - State Research Agency RTC-2017-6401-7.

\section{References}

1. CEN, European Committee for Standarization: EN EN 13306:2017. Maintenance Terminology. European Standard. (2017)

2. Chen, B., Wan, J., Shu, L., Li, P., Mukherjee, M., Yin, B.: Smart factory of industry 4.0: Key technologies, application case, and challenges. IEEE Access 6, 6505-6519 (2018). DOI 10.1109/access.2017.2783682

3. Crespo Marquez, A., Gupta, J.N.: Contemporary maintenance management: process, framework and supporting pillars. Omega 34(3), 313-326 (2006). DOI 10.1016/j.omega.2004.11. 003

4. Ferreira, L.L., Albano, M., Silva, J., Martinho, D., Marreiros, G., di Orio, G., Malo, P., Ferreira, H.: A pilot for proactive maintenance in industry 4.0. In: 2017 IEEE 13th International Workshop on Factory Communication Systems (WFCS). IEEE (2017). DOI 10.1109/wfcs.2017.7991952

5. Goh, K., Tjahjono, B., Baines, T., Subramaniam, S.: A Review of Research in Manufacturing Prognostics. In: 2006 IEEE International Conference on Industrial Informatics, pp. 417-422. IEEE, Singapore (2006). DOI 10.1109/INDIN.2006.275836

6. Hashemian, H.M., Bean, W.C.: State-of-the-Art Predictive Maintenance Techniques*. IEEE Transactions on Instrumentation and Measurement 60(10), 3480-3492 (2011). DOI 10.1109/ TIM.2009.2036347

7. Lee, W.J., Wu, H., Yun, H., Kim, H., Jun, M.B., Sutheralnd, J.W.: Predictive maintenance of machine tool systems using artificial intelligence techniques applied to machine condition data. Procedia CIRP 80, 506-511 (2019)

8. Lu, B., Durocher, D., Stemper, P.: Predictive maintenance techniques. IEEE Industry Applications Magazine 15(6), 52-60 (2009). DOI 10.1109/MIAS.2009.934444

9. Mrugalska, B., Wyrwicka, M.K.: Towards Lean Production in Industry 4.0. Procedia Engineering 182, 466-473 (2017). DOI 10.1016/j.proeng.2017.03.135

10. O'Donoghue, C., Prendergast, J.: Implementation and benefits of introducing a computerised maintenance management system into a textile manufacturing company. Journal of Materials Processing Technology 153, 226-232 (2004)

11. Paolanti, M., Romeo, L., Felicetti, A., Mancini, A., Frontoni, E., Loncarski, J.: Machine learning approach for predictive maintenance in industry 4.0. In: 2018 14th IEEE/ASME International Conference on Mechatronic and Embedded Systems and Applications (MESA). IEEE (2018). DOI 10.1109/mesa.2018.8449150

12. Patil, R.B., Mhamane, D.A., Kothavale, P.B., Kothavale, B.: Fault tree analysis: A case study from machine tool industry. Available at SSRN 3382241 (2018)

13. Potes Ruiz, P.A., Kamsu-Foguem, B., Noyes, D.: Knowledge reuse integrating the collaboration from experts in industrial maintenance management. Knowledge-Based Systems 50, 171-186 (2013). DOI 10.1016/j.knosys.2013.06.005

14. Razmi-Farooji, A., Kropsu-Vehkaperä, H., Härkönen, J., Haapasalo, H.: Advantages and potential challenges of data management in e-maintenance. Journal of Quality in Maintenance Engineering (2019)

15. Rüßmann, M., Lorenz, M., Gerbert, P., Waldner, M., Justus, J., Harnisch, M.: Industry 4.0: The Future of Productivity and Growth in Manufacturing Industries. Boston Consulting Group 9(1), 54-89 (2015)

16. Wan, J., Tang, S., Li, D., Wang, S., Liu, C., Abbas, H., Vasilakos, A.V.: A manufacturing big data solution for active preventive maintenance. IEEE Transactions on Industrial Informatics 13(4), 2039-2047 (2017). DOI 10.1109/tii.2017.2670505 